Revue internationale P.M.E.

Économie et gestion de la petite et moyenne entreprise

Revue

internationale

PME

\title{
Une tontine à double niveau d'enchères
}

\section{Christian E. Rietsch}

Volume 5, numéro 3-4, 1992

URI : https://id.erudit.org/iderudit/1008156ar

DOI : https://doi.org/10.7202/1008156ar

Aller au sommaire du numéro

Éditeur(s)

Presses de l’Université du Québec

ISSN

0776-5436 (imprimé)

1918-9699 (numérique)

Découvrir la revue

Citer cet article

Rietsch, C. E. (1992). Une tontine à double niveau d'enchères. Revue internationale P.M.E., 5(3-4), 89-116. https://doi.org/10.7202/1008156ar

\section{Résumé de l'article}

Nous présentons une tontine d'expatriés camerounais en République centrafricaine. Après avoir vu les règles normales de fonctionnement et les éléments de gestion exceptionnels, nous analysons en détail la technique des enchères des lots principaux et des petits lots. Diverses hypothèses de rationalité financière sont testées, en particulier en ce qui concerne la prise en compte du temps. Il apparaît alors que les participants à la tontine peuvent se regrouper en deux types : les emprunteurs que l'on peut estimer investisseurs à l'extérieur de la tontine et les financiers prêteurs. Les premiers trouvent le capital dont ils ont besoin à un prix compétitif par rapport aux autres possibilités, les seconds font un placement intéressant. Tous bénéficient de la convivialité de la tontine. 


\title{
Une tontine à double niveau d'enchères
}

\author{
Christian E. RIETSCH* \\ Université d'Orléans \\ Université de Niamey
}

\begin{abstract}
RÉSUMÉ
Nous présentons une tontine d'expatriés camerounais en République centrafricaine. Après avoir vu les règles normales de fonctionnement et les élements de gestion exceptionnels, nous analysons en détail la technique des encheres des lots principaux et des petits lots. Diverses hypotheses de rationalité financière sont testées, en particulier en ce qui concerne la prise en compte du temps. II apparait alors que les participants à la tontine peuvent se regrouper en deux types: les emprunteurs que l'on peut estimer investisseurs à l'extérieur de la tontine et les financiers prêteurs. Les premiers trouvent le capital dont ils ont besoin à un prix compétitif par rapport aux autres possibilités, les seconds font un placement intéressant. Tous bénéficient de la convivialité de la tontine.
\end{abstract}

\begin{abstract}
This study presents a tontine which was set up by Cameroonian expatriates in the Central African Republic. It first reviews its standard operating rules as well as exceptional management procedures. It then gives a detailled analysis of bidding techniques for large and small lots. Several hypotheses of financial rationality are tested in particular those which take time into account.

Its participants fall into two categories : borrowers, who can be considered as investors outside of the tontine, and lenders. The former find a ready source of capital at competitive rates and the latter a good return on their money. All derive pleasure from the congenial atmosphere of the tontine.
\end{abstract}

* Christian Rietsch a fait ses études à l'Université de Strasbourg. Docteur ès sciences économiques en 1981 à Nantes, maître de conférences à l'Université d'Orléans en 1985. Il travaille dans le cadre de la coopération française, d'abord comme fiscaliste au Zaïre, puis comme enseignant à l'Université de Bangui (République centrafricaine). Il est actuellement détaché à l'Université de Niamey (Niger). S'intéresse aux problèmes de développement, d'ajustement structurel et de finances informelles. Adresse : B.P. 12442 Niamey, Niger ou s/c M. Bruno Ponson, ESCP, 79, avenue de la République, 75443 Paris Cedex 11, France. 


\section{RESUMEN}

Presentamos una tontina de expatriados del Camerún en la República Centro Africana. Tras haber visto las reglas de funcionamiento normales y los elementos de gestión excepionales, analizamos en detalle la técnica de subasta de los lotes principales y pequeños. Se examinan diversas hipotesis de racionalidad financiera, en particular la relativa al tiempo. Comprobamos así que los participantes de la tontina pueden agruparse en dos tipos: les prestatarios, que pueden ser considerados inversionistas al exterior de la tontina, y los financieros prestamistas. Los primeros encuentran el capital que necesitan a un precio competitivo en relación con las otras oportunidades de las que disponen, los segundos hacen una inversión rentable. Todos salen beneficiados.

\section{Introduction}

On sait qu'une tontine est "un système que se donne un groupe de personnes pour s'obliger collectivement à épargner en vue de jouir tour à tour d'une somme importante ${ }^{l}$ ».

Les tontines sont pour la plupart mutuelles, c'est-à-dire que l'ensemble des cotisations et le montant levé sont égaux : les tontines mutuelles impliquent donc l'absence de prise en compte du temps puisque, pour des cotisations discontinues, la levée unique se passe à un moment différent pour chaque individu et que la levée précoce de la tontine n'est pas considérée comme un avantage sur une levée tardive.

Nous voudrions ici analyser en détail l'organisation, les principes de fonctionnement, la gestion des difficultés et l'intérêt financier d'une tontine complexe à deux niveaux d'enchères ${ }^{2}$ : le premier concerne la levée de l'ensemble des cotisations des membres; le second, un crédit à court terme (un mois).

Mais il y a lieu, avant d'analyser cette tontine, de la présenter.

1. G. Bérard, La mobilisation de l'épargne rurale par les institutions de type coopératif et son impact sur le développement rural: Burkina Faso, Cameroun, Egypte, Nigéria, Rwanda, Togo, Zimbabwe, BIT, Genève, 1985.

2. Cet article constitue une version notablement abrégée et simplifiée de l'analyse complète de cette tontine parue sous les références: C. Rietsch "Une tontine à double niveau d'enchères ", Paris, UREF/AUPELF, Notes de recherche, $n^{\circ}$ 90-5, janv. 1990. 
À l'instigation de quelques hauts fonctionnaires détachés à l'UDEAC ${ }^{3}$ à Bangui (République centrafricaine) s'est créé en 1986 une tontine dénommée : "Tontine de l'élite camerounaise en République centrafricaine ». Le noyau central des membres à l'origine de la tontine est formé de Camerounais d'origine Bamiléké, expatriés et disposant de revenus très élevés par rapport aux fonctionnaires camerounais et sans commune mesure avec ceux de la RCA. S'est greffé à ce noyau central un ensemble de Camerounais expatriés, de professions très diverses : commerçants, artisans, assistant à l'université, personnel travaillant à l'Ambassade du Cameroun... dont les revenus sont plus hétérogènes.

À l'origine du deuxième cycle, 25 membres participent à la tontine. Mais dès la deuxième réunion (tour 3 et 4 ) deux autres membres viennent s'y adjoindre. Enfin, à la troisième réunion, un $28^{\mathrm{e}}$ membre est coopté.

Le fonctionnement du système tontinier est déterminé par les notions de tour et de cycle; s'y ajoute dans le cas présent la notion de réunion. Le tour constitue le moment où un membre bénéficie des cotisations versées par l'ensemble des membres de la tontine ou d'un lot gratuit. Le cycle de la tontine est l'ensemble des tours : il est défini par la durée nécessaire au désintéressement de tous les membres. La tontine que nous présentons en est actuellement à son troisième cycle $e^{4}$. Tous les exemples que nous donnerons dans cet article sont tirés du deuxième cycle dont la première réunion s'est tenue le 5 septembre 1987 et la dernière, le 30 juillet $1988^{5}$. La réunion est l'assemblée des membres de la tontine, tenue ici le dernier samedi de chaque mois ; normalement, chaque réunion correspond à un tour, mais dans le cas présent, pour accélérer les choses, il est organisé lors de chaque réunion deux tours : alors que la cotisation individuelle ou part est de $50000 \mathrm{~F}^{6}$, chaque individu doit donc amener $100000 \mathrm{~F}$ chaque mois. Après une première réunion d'introduction et d'explication des modalités de la tontine, les réunions ordinaires se sont succédées mensuellement comme prévu. Le cycle de la tontine, compte tenu des 28 membres, de 2 tours par réunion et de 4 tours gratuits a nécessité la tenue de

3. Union douanière et économique de l'Afrique centrale : organisme visant une union douanière des États de la sous-région.

4. Ce troisième cycle connaît beaucoup moins de succès que les deux précédents : en effet, des mutations nombreuses et des compressions de personnel ont affecté l'UDEAC ou menacent de l'affecter. Le noyau central des tontiniers qui faisait le succès et inspirait la confiance s'est retiré, ne laissant que 6 membres pour le troisième cycle.

5. Nous sommes redevables à M. Ndefo expert à l'UDEAC et membre du bureau de la tontine, d'une foule de renseignements et d'explications sur le fonctionnement de cette tontine et en particulier de l'obtention de la comptabilité de la tontine.

6. Les sommes sont indiquées en francs CFA ; 1 franc français vaut 50 FCFA. 
12 réunions. À quelques jours près, ce cycle a été bouclé en 11 mois. L'analyse financière se fera donc sur cette durée.

\section{Organisation de la tontine, formalisme et convivialité}

Nous sommes en présence d'une tontine particulièrement structurée, à l'organisation élaborée.

\subsection{L'organisation réglementaire}

Cette tontine dispose d'un nom qui la pose et lui confère une personnalité tant par rapport aux membres que par rapport à l'extérieur. Le nom de « Tontine de l'élite camerounaise en République centrafricaine » est quelque peu pompeux et ridicule - ce dont certains membres sont totalement conscients - mais correspond à quelque chose de réel ${ }^{7}$.

Mais il n'y a pas seulement un nom, il y a surtout un règlement intérieur détaillé et écrit. Ce règlement prévoit l'organisation interne et les modalités du fonctionnement normal ou exceptionnel. Il est probable qu'une tontine gagne de la confiance en établissant un règlement écrit. Il est certain que les procédures d'une tontine à enchères (surtout à deux niveaux) sont plus compliquées que dans le cas d'une tontine mutuelle. De plus, la cooptation d'un nouveau membre entraîne des calculs subtils pour le mettre à égalité avec les anciens. Mais on peut arguer à l'inverse que tous les membres connaissent parfaitement la pratique de la tontine et que les problèmes soulevés par les événements exceptionnels peuvent être réglés selon la coutume. L'existence d'un règlement écrit est donc un gage de sérieux et donne l'assurance du formalisme.

Le règlement prévoit une structuration élaborée de la direction de la tontine, puisque celle-ci est dirigée par « un bureau composé d'un Président, d'un Secrétaire, d'un Trésorier, d'un Commissaire aux Comptes et d'un Censeur » (art. 5a). Chacun des membres du bureau a une fonction bien particulière, précisée par le règlement dans le cadre d'une division des tâches. Les membres du bureau sont élus dans des conditions strictement précisées (art. 5b).

7. Il est de fait que les participants à cette tontine forment une «élite » si l'on entend par là des personnes ayant un niveau culturel élevé, des responsabilités importantes et des revenus sans commune mesure avec le revenu moyen local. 
La structuration de cette tontine apparaît comme relativement complexe, alliant la simple organisation à des fonctions de régulation sociale. En particulier, le contrôle est largement développé, puisqu'un Commissaire aux Comptes vérifie les comptes établis par le trésorier et qu'un secrétaire enregistre les opérations.

\subsection{Le formalisme}

Ce qui frappe le plus l'observateur n'est pas l'organisation de cette tontine, mais son formalisme qui s'exprime dans toute une série de traits :

- Le formalisme des réunions est très important : les réunions commencent «à 16 heures précises ", "le dernier samedi de chaque mois " (art. 1 et 2); un appel est fait et tout retard constaté est sanctionné par une « amende de $2000 \mathrm{~F}$ payable séance tenante » (art. 15), à l'arrivée du retardataire.

- Il est prévu qu'un censeur veille à la discipline lors des séances de la tontine et applique les sanctions prévues par le règlement qui concernent en particulier les « troubles au bon déroulement de la réunion [...] passibles d'une amende de $500 \mathrm{~F}$ payable séance tenante " (art. 16a). Le règlement précise aussi ce qu'il faut entendre par trouble : il s'agit de «bavardages intempestifs, paroles déplacées à l'endroit des membres et tout autre acte ou geste manifeste de troubles ». D'autre part, il est prévu aussi une amende de $10000 \mathrm{~F}$ pour folle enchère (art. 16b).

- Le paiement de la cotisation fait l'objet d'un article à lui seul : le pouvoir libératoire n'est reconnu qu'à deux types de coupures : les billets de $5000 \mathrm{~F}$ et de $10000 \mathrm{~F}$, tout autre billet ou moyen de paiement étant jugé irrecevable (art. 11).

- Les enchères elles-mêmes sont réglementées : il existe une enchère minimum fixée à $5 \%$ du lot principal s'incrémentant ensuite de $1000 \mathrm{~F}$ au minimum (art. 12).

Mais ce formalisme qui frappe l'observateur semble largement compensé par la deuxième caractéristique de la tontine : sa convivialité.

\subsection{La convivialité}

La tontine se réunit chez l'adjudicataire du mois précédent (art. 2); s'il y a plusieurs adjudicataires - et ce sera le cas ici, puisque pour accélérer le cycle, il y en aura deux à chaque séance - ceux-ci devront s'entendre séance tenante pour décider qui recevra la tontine le mois suivant, étant entendu qu'en cas 
d'empêchement ce serait systématiquement à l'autre adjudicataire de recevoir (art. 3a). De toute façon, en cas d'empêchement, celui qui doit recevoir doit prendre toutes les dispositions qui s'imposent et en informer le président au moins 3 jours avant la date de la réunion (art. $3 b$ ).

Le membre qui reçoit la tontine organise une réception. À ce titre, il perçoit une somme de $5000 \mathrm{~F}$ par membre quel que soit le nombre de parts de celui-ci (art. 20) : la collecte pour frais de réception est liée au coût individuel de la réception et non au nombre de parts financières. Bien que la vie soit très chère à Bangui, cette somme permet d'organiser une véritable fête où sont servis les mets les plus prisés par les membres (en particulier, du poulet et du ndolé) arrosés copieusement. Il est considéré de bon ton pour celui qui reçoit la tontine de ne pas lésiner sur la nourriture et les boissons et de ne pas chercher à économiser une fraction des sommes reçues. En général, les épouses des membres rivalisent d'adresse pour préparer et présenter le plus grand nombre de mets, de la meilleure qualité.

Ce serait un grand tort de négliger le côté festivité de la tontine pour ne considérer que l'aspect financier : la fête permet de se retrouver, de tisser des liens, de se détendre. En même temps, elle permet littéralement de «s'empiffrer » : la nourriture est tellement abondante et la conscience que de toute façon on a payé est telle que la réaction de nombreux participants est d'en profiter et de « se remplir le ventre ${ }^{8}$.

Mais la convivialité ne se limite pas aux seuls repas pris en commun à l'issue d'une réunion de la tontine. Elle se manifeste aussi pour la nouvelle année : l'ensemble du groupe se retrouve pour une grande fête (pour laquelle chacun a cotisé $15000 \mathrm{~F}$ ). Enfin, on hésite à qualifier de conviviale ou de formaliste la réception que la tontine offre à l'ambassadeur du Cameroun et pour laquelle chaque membre cotise $20000 \mathrm{~F}$; ce qui est certain, c'est que la réception consacre la tontine face à l'extérieur et lui confère un brevet d'honorabilité.

\section{Le fonctionnement de la tontine}

Il y a lieu de distinguer la gestion normale de la tontine de celle des difficultés.

8. Il convient aussi d'être conscient que les sommes collectées sont considérables : pour certains, les cotisations à la tontine et aux frais de réception épuisent littéralement toute l'encaisse : le lendemain, il n'y a littéralement rien à mettre dans l'assiette. La réception est donc l'occasion de prendre un peu d'avance, de consommer avant de devoir se restreindre ; pour ceux-ci, la fête est d'autant plus prisée. 


\subsection{Les principes de la gestion quotidienne}

La tontine que nous décrivons est à deux niveaux d'enchères : il existe une enchère pour le lot principal et une autre enchère pour ce que l'on appelle les «petits lots »; éventuellement, le lot principal pourra être mis en adjudication « gratuitement » quand le montant des petits lots dépasse un certain seuil.

\subsubsection{Le lot principal}

Le principe est simple : les membres de la tontine, ici 28 personnes, cotisent chacune une somme de $50000 \mathrm{~F}$. L'ensemble des cotisations constitue le lot principal : celui-ci est donc de $1400000 \mathrm{~F}$ dans notre cas. La question est alors celle de l'attribution du lot principal. Au contraire de la tontine mutuelle, où l'ordre des levées est généralement établi d'un commun accord et susceptible d'aménagements, l'attribution du lot principal dépend ici d'enchères. Lors d'un tour, toute personne qui n'a pas encore été adjudicataire peut enchérir et l'individu qui offre enchère la plus forte lève le lot principal et est dit adjudicataire. Il récupère la somme collectée, déduction faite des enchères. Par exemple, au $8^{\mathrm{e}}$ tour, l'adjudicataire est $1^{\prime}$ individu $\mathrm{n}^{\circ} 3$ qui a fait monter les enchères à $216000 \mathrm{~F}$ : il touche la somme de $1184000 \mathrm{~F}$ (soit $1400000 \mathrm{~F}-216000 \mathrm{~F}$ ).

Il convient de noter que :

- Un individu peut participer à la tontine pour plusieurs parts. Dans notre cas, les individus $n^{\circ} 3,5$ et 15 ont pris deux parts : à chaque tour, ils devaient donc cotiser $100000 \mathrm{~F}$ par part, c'est-à-dire $200000 \mathrm{~F}$ chaque mois.

- La mise à prix du lot principal est de $5 \%$ au minimum : il en coûte donc au moins $70000 \mathrm{~F}$ pour lever le lot principal; dans le cas où il n'y a pas d'enchérisseur, le preneur est tiré au sort parmi les membres qui n'ont pas encore levé le lot principal : c'est ce qui est arrivé par exemple lors des derniers tours où tous les adjudicataires ont été tirés au sort (tours 24 à 28 ) : chacun de ces adjudicataires a subi un prélèvement de $70000 \mathrm{~F}$ (soit $5 \%$ de $1400000 \mathrm{~F}$, versés à la caisse) et n'a touché que $1330000 \mathrm{~F}$.

- Un membre coopté par la tontine ne peut être adjudicataire du lot principal avant la moitié des membres l'ayant coopté (cooptation lors du début du cycle) ou la moitié des membres n'ayant pas encore levé le lot principal (cooptation au cours du cycle). En ce qui concerne les individus 26 et 27 , les dispositions réglementaires se sont appliquées sans problème, puisqu'ils n'ont levé qu'au $24^{e}$ et $26^{e}$ tour. Mais pour ce qui concerne $1^{\prime}$ individu $n^{\circ} 28$, coopté au début du $6^{\mathrm{e}}$ tour, ces dispositions ne se sont pas appliquées puisque cet individu a levé la tontine 
au $11^{\mathrm{e}}$ tour, car « tout simplement le $\mathrm{n}^{\circ} 28$ est solvable et personne n'a fait attention à cet article » nous dit notre informateur : la confiance et le crédit personnel l'ont donc largement emporté sur le strict respect du règlement.

- La remise du lot principal nécessite pour l'adjudicataire de présenter un avaliste. Celui-ci accepte sa fonction et sert de garantie à l'adjudicataire dans les conditions ci-dessous. Si l'adjudicataire ne peut pas présenter un avaliste, «il est coupable de folle enchère et le lot est remis en vente $»($ art. 13).

\subsubsection{Les petits lots}

Formellement, l'existence de petits lots découle de l'article 14 du règlement qui prévoit que "le produit de la vente aux enchères ainsi que les produits découlant des pénalités sont constitués en petits lots cessibles aux enchères, dans les mêmes conditions que le lot principal, à tout membre de la tontine, entendu que le montant des intérêts est prélevé séance tenante »?.

L'idée est la suivante : si à la suite des opérations de la tontine, il existe une encaisse, celle-ci peut être prêtée d'un mois sur l'autre, le bénéficiaire du prêt étant déterminé au moyen d'enchères. De façon pratique, pour ne pas constituer des sommes trop importantes, difficiles à gérer et à l'utilisation problématique, on fractionne le fonds en caisse en une série de lots (d'où le nom) constitués de sommes rondes $(100000 \mathrm{~F}, 50000 \mathrm{~F}, \ldots)$ ) proposées aux enchères. On note que :

- celui qui bénéficie du petit lot se voit remettre la somme déduction faite du montant des enchères ;

- le petit lot emprunté est remboursable en principal le mois suivant ;

- chaque membre peut enchérir les petits lots de façon totalement indépendante du lot principal sans qu'un désintéressement n'intervienne ;

- un membre qui le désire peut prendre plusieurs petits lots le même jour.

9. En fait il convient d'ajouter à cette procédure simple les remarques suivantes :

- Il convient d'augmenter le fonds du reste en caisse du cycle précédent ou du tour précédent. Dans le cas présent, la tontine a hérité du cycle précédent la somme de $125000 \mathrm{~F}$ et d'un fonds de caisse de $7770 \mathrm{~F}$.

- Il ne faut évidemment pas oublier le remboursement des petits lots du tour précédent qui peuvent à nouveau être prêtés. 
Par exemple, lors de la première réunion, les petits lots ont été distribués aux conditions suivantes :

TABleau 1

Petits lots de la première réunion

\begin{tabular}{cccccc}
\hline $\begin{array}{c}\text { Numéro } \\
\text { du lot }\end{array}$ & $\begin{array}{c}\text { Montant brut du } \\
\text { petit lot (FCFA) }\end{array}$ & $\begin{array}{c}\text { Intérêt payé } \\
\text { (FCFA) }\end{array}$ & $\begin{array}{c}\text { Montant net du } \\
\text { petit lot (FCFA) }\end{array}$ & $\begin{array}{c}\text { Taux d'intérêt } \\
\text { mensuel ( \%) }\end{array}$ & $\begin{array}{c}\text { Numéro } \\
\text { d'adjudicataire }\end{array}$ \\
\hline 1 & 125000 & 25000 & 100000 & $25,00 \%$ & 25 \\
2 & 100000 & 16000 & 84000 & $19,05 \%$ & 23 \\
3 & 100000 & 13000 & 87000 & $14,94 \%$ & 12 \\
4 & 100000 & 15000 & 85000 & $17,65 \%$ & 12 \\
5 & 100000 & 13000 & 87000 & $14,94 \%$ & 23 \\
6 & 100000 & 12000 & 88000 & $13,64 \%$ & 3 \\
7 & 50000 & 11000 & 39000 & $28,21 \%$ & 9 \\
8 & 40000 & 7000 & 33000 & $21,21 \%$ & 13 \\
9 & 5000 & 200 & 4800 & $4,17 \%$ & 9 \\
10 & 100000 & 6000 & 94000 & $6,38 \%$ & 9 \\
\hline
\end{tabular}

On voit dans cet exemple que le premier lot de $125000 \mathrm{~F}$ a été levé à l'aide d'une enchère de $25000 \mathrm{~F}$ : l'adjudicataire, l'individu $\mathrm{n}^{\circ} 25$, a donc touché la somme de $100000 \mathrm{~F}$. Lors de la réunion suivante, un mois plus tard, cet individu a dû rembourser l'intégralité du petit lot, soit $125000 \mathrm{~F}$. Il a donc bénéficié d'un crédit au coût de $25 \%$ pour le mois. On notera l'importance des taux d'intérêt qui s'échelonnent, dans cet exemple, entre $4 \%$ et $25 \%$ par mois ; sur l'ensemble du cycle, on a pu voir des taux allant jusqu'à $42,9 \%$ d'intérêt par mois ${ }^{10}$.

\subsubsection{Les tours gratuits}

Le tour gratuit n'est pas expliqué par le règlement mais découle de la pratique tout en constituant l'un des plus grands intérêts de la tontine. Le principe en est le suivant : « si au cours d'une séance on constate que le montant des intérêts est égal à la valeur d'un lot principal, on vend plutôt le lot principal pour

10. De tels chiffres peuvent paraître extraordinaires à quelqu'un de non averti. Il convient toutefois d'être conscient des faits suivants : à Bangui, le taux d'intérêt sur un mois, entre amis est de $50 \%$; il est de $100 \%$ entre simples connaissances; d'autre part, ces taux d'intérêt ne concernent que de petites sommes : $1000 \mathrm{~F}, 5000 \mathrm{~F}$, $30000 \mathrm{~F}$ au maximum ; il est quasi impossible d'emprunter des sommes plus importantes à travers de simples particuliers, connaissances, amis et parents : on en déduit a contrario que les membres de la tontine ont bien de la chance de pouvoir emprunter de fortes sommes à des taux considérés comme relativement faibles. 
désintéresser un membre qui n'a pas été déjà adjudicataire ${ }^{11}$ » alors que "normalement, c'est à la fin du cycle que l'on partage les intérêts éventuels. Mais en réalité les intérêts sont partagés entre les membres dès que le montant atteint la valeur du lot principal [...] Dans le tour gratuit, tout se passe comme si on avait donné à chacun la valeur d'une part » et que chacun «l'utilise pour cotiser à nouveau ». L'avantage souligné par les participants est de raccourcir le cycle : avec 28 membres, deux tours par réunion et une réunion par mois, il faut normalement 13 mois pour boucler le cycle; ici, compte tenu des 4 tours gratuits, il n'en a fallu que 11 .

Techniquement, le trésorier regarde ce dont il dispose en caisse après que les tours normaux prévus lors de la réunion ont eu lieu, produisant chacun une entrée d'enchères en caisse, après que les bénéficiaires de petit lot de la précédente réunion ont remboursés leurs dus, les retardataires et perturbateurs payés leurs amendes, etc. Si ce qui reste à ce moment-là est supérieur au montant du lot principal, on décide de l'attribution du lot gratuit : celui-ci est mis aux enchères comme un lot principal, mais sans que quiconque ait besoin de cotiser.

Par exemple, aux $16^{\mathrm{e}}$ et $17^{\mathrm{e}}$ tours, il y avait 28 participants, ce qui a permis deux adjudications de lots principaux de $1400000 \mathrm{~F}$. Le solde en caisse du tour précédent $(7470 \mathrm{~F})$ augmenté des remboursements de petits lots $(1525000 \mathrm{~F}$ ) et des enchères concernant les deux lots principaux (soit $78000 \mathrm{~F}$ et $75000 \mathrm{~F}$ ) permet de disposer de $1685470 \mathrm{~F}$, une somme supérieure à la valeur d'un lot principal (1400 000 F) : on organise un tour gratuit qui rapporte $71000 \mathrm{~F}$ d'enchères. À ce moment, il reste en caisse : $1685470 \mathrm{~F}$ $1400000 \mathrm{~F}+71000 \mathrm{~F}$, soit $356470 \mathrm{~F}$. Cette somme est prêtée en une série de 6 petits lots et il reste une encaisse finale de $970 \mathrm{~F}$.

\subsection{La gestion des difficultés}

La gestion courante de la tontine est simple. Mais si des événements exceptionnels surviennent, il faut que ces cas puissent être réglés sans léser les participants.

\subsubsection{Les défaillances}

Tout membre qui ne peut payer séance tenante sa part ou qui ne peut rembourser le petit lot dans les conditions prévues est déclaré défaillant. Celui qui est

11. Lettre du 16 juillet 1988 de M. Ndefo à l'auteur. 
déclaré défaillant a un mois pour rembourser sa dette majorée d'une pénalité de $25 \%$ s'il n'a pas encore été adjudicataire et de $50 \%$ dans le cas contraire ${ }^{12}$.

Mais le règlement prévoit une défaillance prolongée où l'on applique l'article 19 selon deux modalités :

- Si le défaillant a bénéficié du lot principal, il existe peu de moyens de pression financiers sur lui : aussi, son avaliste devra payer à sa place le principal et les pénalités. Puis « l'avaliste avec le concours du bureau poursuit le débitant principal ». Mais si l'avaliste est dans l'incapacité de payer «sa ou ses parts sont saisies à concurrence du montant pour lequel il a donné son aval » (art. 19a).

- Si le défaillant n'a pas encore bénéficié du lot principal, il est exclu et « peut attendre la fin du cycle pour récupérer les fonds effectivement versés par lui, majorés des tours gratuits survenus avant sa défaillance, déduction faite des pénalités dues »; mais le règlement prévoit aussi, dans la même phrase, que s'il ne peut attendre la fin du cycle « il lui est restitué intégralement les sommes qu'il aura effectivement versées, déduction faite des pénalités ».

\subsubsection{L'adhésion de nouveaux membres}

Les nouveaux membres sont admis dans la tontine par cooptation des anciens. Cette cooptation peut se faire selon deux modalités totalement différentes : au début d'un cycle ou au cours d'un cycle; le premier cas ne soulève aucune difficulté, alors que le second en est hérissé.

La cooptation en début de cycle est prévue à l'article 23, lequel précise que « les nouveaux membres sont cooptés par ceux ayant participé au cycle précédent ». Le membre ainsi coopté ne peut être adjudicataire du lot principal avant la moitié des participants au cycle précédent. Ces deux dispositions donnent aux membres de la tontine qui ont fait leur preuve la possibilité de limiter l'accès à la tontine aux personnes jugées de confiance et fournissent la garantie que les nouveaux arrivants ne vont pas profiter de la tontine pour frauder et être défaillant : la première moitié du cycle constitue donc une période probatoire durant laquelle le membre coopté cotise, c'est-à-dire s'engage financièrement auprès des anciens qui disposent de moyens de pression. Aucun problème financier ne se pose et il revient au président et au trésorier de veiller à ce que

12. Cette différence entre les taux de pénalité est particulièrement significative : quand le groupe dispose d'un moyen de pression, la pénalité est de $25 \%$; quand il n'en dispose pas directement, elle est de $50 \%$. Ce taux, dans les conditions locales, est très exactement celui d'un petit prêt à un ami : on appréciera ! 
le nouveau membre ne soit pas adjudicataire avant la moitié des membres qui ont participé au cycle précédent.

La cooptation en cours de cycle limite, dans des conditions similaires, les possibilités d'être adjudicataire du lot principal. Évidemment, le membre coopté doit se mettre en règle d'une part avec l'ensemble des membres, et d'autre part, avec les membres adjudicataires avant sa cooptation. L'apurement de son compte fait l'objet de prescriptions très détaillées qui se différencient selon qu'il y a eu des tours gratuits ou non.

\subsubsection{Le retrait de la tontine}

Tout membre de la tontine peut se retirer au cours du cycle (art. 26a). Évidemment, il lui revient de se mettre en règle avec les autres membres de la tontine. Deux procédures sont envisagées selon que celui qui se retire a déjà été ou non adjudicataire.

\section{L'analyse financière de la tontine}

Après avoir étudié les modalités de l'organisation de la tontine et les techniques de la gestion courante et des difficultés, il nous faut maintenant aborder l'analyse financière. Pour ce faire, nous allons d'abord considérer les levées du lot principal, puis la gestion financière des petits lots ; enfin, nous intégrerons les deux opérations ainsi que les coûts de la convivialité pour mesurer l'intérêt individuel de la participation à la tontine.

\subsection{Analyse financière du lot principal}

L'hypothèse de base est que l'on verra apparaître une baisse des enchères du lot principal à mesure que le cycle avance. Trois explications, qui méritent d'être testées, concourent à expliquer ce phénomène :

- Les enchères forment une mesure de l'urgence des besoins des divers participants, les besoins les plus urgents sont satisfaits aux prix les plus élevés.

- Les enchères constituent le coût du crédit; or, à chaque nouvelle réunion, la durée du crédit obtenu est raccourcie d'un mois : il est donc naturel que le coût d'un crédit plus court soit inférieur au coût d'un crédit plus long. 
- La pression de la demande : le montant atteint par les enchères est fonction du nombre de personnes susceptibles d'enchérir : or, celui-ci diminue à mesure qu'avance le cycle, réduisant d'autant la pression de la demande.

Les trois raisons que nous venons de citer ne sont pas indépendantes.

\subsubsection{L'hypothèse des enchères du lot principal en tant que mesure de l'urgence des besoins}

L'étude de cette hypothèse peut se mener selon deux points de vue : par réunion ou par cycle.

\section{- Sur tout le cycle}

On peut penser que les besoins les plus urgents s'expriment les premiers, donc lors des premières réunions, et les besoins les moins urgents lors des dernières réunions. Si tel est le cas, on doit trouver des enchères élevées lors des premières réunions et des enchères plus faibles lors des réunions suivantes; mais ceci présume que les besoins soient connus et planifiés sur tout le cycle; on peut aussi supposer que s'il survient un événement exceptionnel, cela se traduit par un besoin de fonds urgent : dans un tel cas, sur un trend descendant des enchères, une hausse brutale lors d'une réunion apparaît. C'est exactement ce que l'on note sur le tableau suivant, lequel illustre la distribution du coût des levées selon le rang des réunions.

TABLEAU 2

Coût de la levée des lots principaux en fonction du rang des réunions

\begin{tabular}{lrrrrrrrrrrrr}
\hline Rang de la réunion & $\mathbf{1}$ & $\mathbf{2}$ & $\mathbf{3}$ & $\mathbf{4}$ & $\mathbf{5}$ & $\mathbf{6}$ & $\mathbf{7}$ & $\mathbf{8}$ & $\mathbf{9}$ & $\mathbf{1 0}$ & $\mathbf{1 1}$ & $\mathbf{1 2}$ \\
\hline Enchères & 260 & 370 & 336 & 155 & 210 & 75 & 78 & 70 & 70 & 75 & 70 & 70 \\
(milliers de FCFA) & 320 & 375 & 311 & 120 & 200 & 70 & 75 & 125 & 70 & 91 & 70 & 70 \\
& & 340 & 216 & & & & 71 & & & & & 70 \\
Enchères en \% du lot & 26,26 & 37,76 & 31,58 & 12,45 & 17,65 & 5,67 & 5,9 & 5,26 & 5,67 & 5,26 & 5,26 & \\
levé ou coût relatif & 34,41 & 38,46 & 28,56 & 9,38 & 16,67 & 5,26 & 5,67 & 9,8 & 5,26 & 6,91 & 5,26 & 5,26 \\
de la levée & & 33,66 & 18,24 & & & & 5,34 & & & & & 5,26 \\
\hline
\end{tabular}

Le coût de la levée du lot principal varie entre un minimum de $70000 \mathrm{~F}$ (enchère minimum) et un maximum de $375000 \mathrm{~F}$; mais comme ces sommes sont relatives à un nombre de cotisants différent (à cause de la cooptation au cours du cycle), il est plus judicieux de travailler sur le montant des enchères que sur la somme nette levée. Dans ces conditions, le coût relatif de la levée varie entre $5,26 \%$ et $38,46 \%$ du montant perçu : visiblement, les premières 
réunions génèrent des coûts relatifs plus élevés que les dernières : un test de Mann et Whithney confirme cette supériorité à $1 \%{ }^{13}$. Notre hypothèse de baisse des enchères à mesure que les besoins urgents sont comblés semble donc être corroborée.

\section{- $\quad$ À l'intérieur de chaque réunion}

L'urgence relative des besoins doit s'exprimer par des enchères plus élevées lors du premier tour que lors du second ou du troisième.

TABLEAU 3

\section{Enchères à l'intérieur des réunions}

\begin{tabular}{cccc}
\hline \multirow{2}{*}{$\begin{array}{c}\text { Rang de } \\
\text { la réunion }\end{array}$} & \multicolumn{3}{c}{ Enchères de la réunion en \% du lot principal levé } \\
\cline { 2 - 4 } & lors du premier tour de la réunion & lors du ou des tours suivants \\
\hline 1 & 26,26 & 34,31 & \\
2 & 37,76 & 38,46 & 33,67 \\
3 & 31,58 & 28,56 & 18,24 \\
4 & 12,45 & 9,38 & \\
5 & 17,65 & 16,67 & \\
6 & 5,67 & 5,26 & \\
7 & 5,9 & 5,66 & \\
8 & 5,26 & 9,8 & \\
9 & 5,26 & 5,26 & \\
10 & 5,26 & 6,95 & \\
11 & 5,26 & 5,26 & 5,26 \\
12 & 5,26 & 5,26 & \\
\hline
\end{tabular}

Un test de signe sur les taux d'enchères lors du premier tour d'une réunion et le ou les tours suivants ne permet pas de conclure à une différence significative ${ }^{14}$. L'inégale urgence des besoins ne semble donc pas se manifester à l'intérieur de chaque réunion.

\subsubsection{L'hypothèse de la baisse des enchères liée à la durée du crédit}

Le montant des enchères rapporté au montant net levé donne un taux brut d'intérêt. Mais de réunion en réunion, la durée sur laquelle porte le crédit ainsi

13. Le test de Mann et Whithney possède l'avantage sur le test $t$ de Student de ne pas nécessiter l'hypothèse d'égalité des variances (qui, ici, n'est pas vérifiée).

14. Le risque de première espèce est de $19,4 \%$. 
obtenu diminue d'un mois tandis que la durée de la cotisation augmente d'autant : l'analyse du coût du crédit doit en conséquence se faire par réunion. Il est alors intéressant de voir le rapport entre la durée du crédit et son coût. Nous obtenons le graphique suivant, d'après les données du tableau ci-dessus :

\section{GRAPHIQUE 1}

\section{Enchères en \% du lot levé en fonction du rang de la réunion}

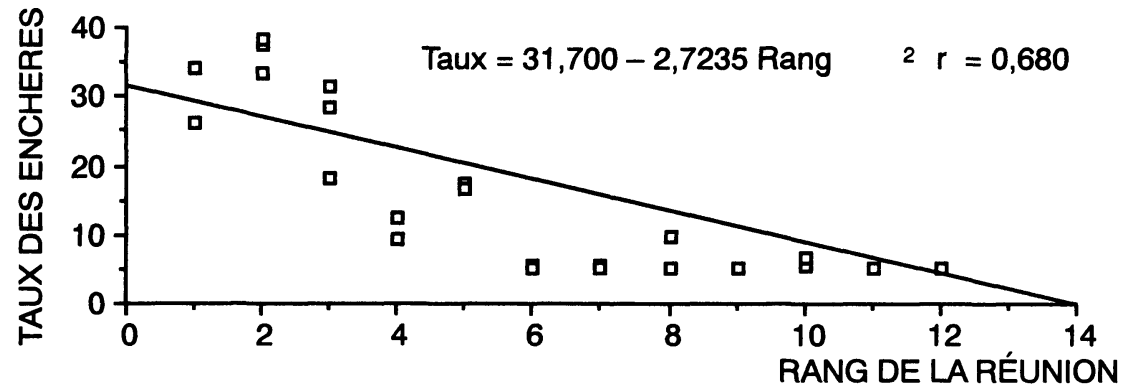

Sur l'ensemble du cycle, l'ajustement le plus « parlant » est :

$$
t=31,7-2,7235 R \quad r^{2}=0,68
$$

où $t$ est le taux relatif des enchères et $R$ est le rang de la réunion.

Cette équation d'ajustement, bien que non la meilleure ${ }^{15}$, nous dit que le taux moyen d'enchère baisse à chaque réunion successive d'environ $2,7 \%$. Le taux apparent d'intérêt est donc de 2,7\% par mois.

Les premiers individus à lever le lot principal sont prêts à payer beaucoup pour disposer immédiatement d'argent en vue d'une utilisation longue qui les intéresse : nous les appelons « opérateurs ». Les derniers à lever le lot principal attendent que d'autres s'engagent financièrement : ils prêtent de l'argent et en attendent une rémunération; nous les appelons « financiers ». Cette distinction sera amplement utilisée plus loin.

\subsubsection{L'hypothèse de la baisse des enchères du lot principal liée à la pression de la demande}

Au lieu de nous intéresser aux déterminants économiques des enchères du lot principal, nous pouvons aussi nous contenter d'une approche naïve en faisant

15. Sur l'ensemble du cycle, le meilleur ajustement obtenu est : $t=R^{-0,949}, 49,75 r=$ 0,895 . 
l'hypothèse que le niveau atteint par les enchères est fonction du nombre de personnes susceptibles d'enchérir.

Là encore, deux solutions peuvent être envisagées :

\section{- L'approche a priori}

A priori, chaque personne pèse d'un même poids sur la demande de levée. Les enchères peuvent donc être envisagées comme une fonction du nombre (décroissant) des personnes susceptibles d'enchérir. Évidemment, deux cas sont à considérer selon que le règlement est appliqué ou non en ce qui concerne les membres cooptés. La pression de la demande explique le taux des enchères $(t)$ de la façon suivante :

- en respectant le règlement, c'est-à-dire en tenant compte du nombre de personnes susceptibles d'enchérir $\left(X_{2}\right)$, en particulier des droits à enchérir des membres cooptés en cours de cycle, nous obtenons ${ }^{16}$ :

$$
\begin{aligned}
t=-4,46+1,45 X_{2} & r^{2}=0,72 \\
(0,18) & n=28
\end{aligned}
$$

- sans égard pour le règlement, c'est-à-dire en tenant compte du nombre total d'adjudicataires potentiels (que nous désignons par $X_{1}$ ) :

$$
\begin{aligned}
t=-3,68+1,26 X_{1} & r^{2}=0,68 \\
(0,17) & n=28
\end{aligned}
$$

Dans les deux cas, chaque individu supplémentaire susceptible d'enchérir pousse le coût relatif de la levée vers le haut d'un peu plus d'un point de pourcentage.

\section{- L'approche a posteriori}

A posteriori, nous connaissons la nature des intervenants : nous savons qui sont les opérateurs et qui sont les financiers. En introduisant une variable muette $\left(X_{3}\right)$ valant 1 pour les financiers et 0 pour les opérateurs, on obtient, selon que le règlement est respecté ou non :

- eu égard au règlement :

$$
\begin{array}{cl}
t=-17,83+2,22 X_{2}+23,18 X_{3}-2,17 X_{2} X_{3} & r^{2}=0,88 \\
(0,36)(7,57) & (0,46) \quad n=28
\end{array}
$$

ce qui s'écrit pour les opérateurs : $\quad t=-17,83+2,22 X_{2}$

et pour les financiers : $\quad t=5,25+0,05 X_{2}$

16. Les écarts types des coefficients sont indiqués entre parenthèses sous les coefficients ; ils sont tous significatifs à $1 \%$ au moins. 
- sans égards pour le règlement :

$$
\begin{aligned}
& t=-41,76+3,09 X_{1}+47,27 X_{3}-3,06 X_{1} X_{3} \quad r^{2}=0,9 \\
& (0,43) \quad(9,69) \quad(0,48) \quad n=28
\end{aligned}
$$

d'où l'équation du comportement des opérateurs : $t=-41,76+3,09 X_{1}$ et celle des financiers :$$
t=5,51+0,05 X_{1}
$$

La pression de la demande détermine largement le niveau atteint par les enchères des lots principaux. La présence de chaque opérateur susceptible d'enchérir augmente le coût relatif de la levée de 2,2 points de pourcentage si l'on tient compte du règlement, de plus de 3 points si l'on n'en tient pas compte. Entre opérateurs, la concurrence est très vive pour le lot principal, alors que pour les financiers, ce n'est pas le cas : ils attendent que les opérateurs déterminent au moyen d'enchères qui va lever le lot principal et leur taux d'enchère est quasi indépendant du nombre d'adjudicataires potentiels.

\section{Conclusion}

La levée des lots principaux répond en grande partie aux différentes hypothèses de base de la rationalité financière et économique. En effet, on peut montrer que le niveau des enchères baisse au cours du cycle, phénomène qui peut être expliqué de diverses façons ; mais d'autre part, à l'intérieur d'une réunion, il n'existe pas de différence significative selon l'urgence présumée des besoins, ce qui laisse planer au moins un doute sur la rationalité.

\subsection{Analyse financière des petits lots}

\subsubsection{Les emprunteurs}

Certains membres de la tontine ne demandent jamais de crédit à court terme ; d'autres y recourent occasionnellement une ou deux fois au cours du cycle; d'autres, enfin, ont souvent besoin de crédits, voire chaque mois, tellement leur manque de trésorerie est important. Globalement, 108 prêts à un mois ont été distribués, soit en moyenne 9,8 prêts par réunion et 4,3 prêts par membre : en moyenne, un individu vivait avec un emprunt d'un mois durant près de $40 \%$ du cycle. Une très forte concentration des prêts apparaît : 8 membres, soit $32 \%$ de l'effectif n'ont pas besoin de prêts ; les 5 membres ayant le plus de prêts ont, dans l'ensemble, bénéficié de $62 \%$ des prêts et l'individu qui en a le plus épuise $22 \%$ des prêts. Considérons alors la nature des emprunteurs de petits lots. On découvre que les opérateurs ont un comportement totalement différent de celui des financiers, comme en témoigne le tableau suivant :

\section{TABLEAU 4}


Emprunt de petits lots en fonction du comportement lors de la levée du lot principal

\begin{tabular}{lccc}
\hline & $\begin{array}{c}\text { Nombre moyen } \\
\text { d'emprunts }\end{array}$ & $\begin{array}{c}\text { Écart type du } \\
\text { nombre d'emprunts }\end{array}$ & $\begin{array}{c}\text { Nombre } \\
\text { d'observations }\end{array}$ \\
\hline Opérateurs & 7,60 & 7,26 & 10 \\
Financiers & 2,13 & 2,97 & 15 \\
\hline
\end{tabular}

\subsubsection{Les sommes empruntées}

Celles-ci vont de petits appoints mensuels exceptionnels (46 $000 \mathrm{~F}, 95000 \mathrm{~F} .$. ) à des sommes importantes et régulières, le record étant détenu par l'individu $\mathrm{n}^{\circ} 12$ qui emprunte $1861500 \mathrm{~F}$ durant le cycle en 24 petits lots.

TABLEAU 5

Distribution des sommes empruntées

\begin{tabular}{cc}
\hline $\begin{array}{c}\text { Sommes empruntées } \\
\text { (milliers de FCFA) }\end{array}$ & Nombre d'observations \\
\hline 0 à moins de 100 & 4 \\
100 à moins de 200 & 2 \\
200 à moins de 400 & 3 \\
400 à moins de 600 & 4 \\
600 à moins de 800 & 1 \\
800 à moins de 1200 & 2 \\
1200 à moins de 1600 & 0 \\
1600 à moins de 2000 & 1 \\
\hline
\end{tabular}

Sur l'ensemble du cycle, plus de 8 millions ont été empruntés à très court terme, soit plus de $735000 \mathrm{~F}$ par réunion. Un seul individu emprunte $23 \%$ de cette somme et les 4 plus importants emprunteurs en cumulent $56 \%$. Quand on étudie la combinaison entre le nombre d'emprunts et la somme empruntée, on obtient un coefficient de corrélation de rang de 0,984 , ce qui signifie que ceux qui empruntent le plus souvent sont aussi les emprunteurs les plus importants en ce qui concerne la somme totale empruntée.

\subsubsection{Le montant des intérêts}

Les intérêts des petits lots s'élèvent à $965000 \mathrm{~F}$; rapportés à la somme totale empruntée $(8084000 \mathrm{~F})$, les intérêts sont de $12 \%$ par mois en moyenne. La somme totale d'intérêts payés par un emprunteur va de $4000 \mathrm{~F}$ pour un em- 
prunt à $226500 \mathrm{~F}$ pour 24 emprunts, la moyenne parmi les emprunteurs étant de $56700 \mathrm{~F}$ d'intérêt. Une corrélation de rang entre le nombre d'emprunts et la somme d'intérêts payés ressort à 0,934 , soit moins que la corrélation précédente : cela suggère que certains emprunteurs gèrent mieux que d'autres leurs emprunts.

\subsubsection{Taux d'intérêt des petits lots et somme empruntée}

Une des caractéristiques remarquables de l'emprunt des petits lots est que le taux d'intérêt moyen payé par un individu est très faiblement corrélé avec la somme empruntée, que cette corrélation soit calculée entre les sommes et le taux moyen $(r=0,12)$ ou plus simplement entre les rangs $(r=0,41)$. On en déduit que certains individus, pressés par le besoin de crédit, enchérissent très fortement, même pour obtenir une petite somme (par exemple, l'individu ${ }^{\circ} 2$ obtient $163000 \mathrm{~F}$ de crédit à $22 \%$ en moyenne par mois); d'autres, pour des sommes très semblables, paient beaucoup moins ; enfin, les très gros emprunteurs ne s'en tirent pas si mal : le plus gros emprunte à $12,2 \%$ en moyenne, alors qu'il absorbe $22 \%$ des emprunts. On peut déduire de ces chiffres l'idée de stratégies personnelles : certains empruntent systématiquement; d'autres très rarement et seulement quand le coût du crédit leur paraît abordable ; par contre, en cas de besoin impérieux, quelques-uns n'hésiteront pas à payer très cher l'accès au crédit.

\subsubsection{La structure des taux d'intérêt des petits lots}

On peut poser une double hypothèse concernant le taux d'intérêt des petits lots :

- Il n'existe pas de raison a priori pour que le taux d'intérêt moyen au cours d'une réunion soit différent selon que l'on se trouve au début ou à la fin du cycle : en effet, le crédit porte toujours sur une période d'un mois et à chaque fois le même nombre d'individus est susceptible d'enchérir.

- Lors d'une réunion donnée, les besoins de crédit à court terme seront satisfaits dans l'ordre décroissant et les enchères à l'intérieur d'une réunion donnée diminuent quand le rang du petit lot crôit.

Les chiffres sont les suivants :

\section{TABLEAU 6}


Intérêts, sommes prêtées et taux d'intérêt moyen par réunion

\begin{tabular}{lrrrrrrrrrrrr}
\hline Rang de la réunion & $\mathbf{1}$ & $\mathbf{2}$ & $\mathbf{3}$ & $\mathbf{4}$ & $\mathbf{5}$ & $\mathbf{6}$ & $\mathbf{7}$ & $\mathbf{8}$ & $\mathbf{9}$ & $\mathbf{1 0}$ & $\mathbf{1 1}$ & ToTAL \\
\hline $\begin{array}{l}\text { Intérêts précomptés } \\
\text { (milliers de FCFA) }\end{array}$ & 106,2 & 112 & 64,5 & 96,5 & 182 & 63,5 & 43,8 & 88 & 57 & 94 & 57,5 & 96,5 \\
$\begin{array}{l}\text { Sommes prêtées } \\
\text { (milliers de FCFA) }\end{array}$ & 674 & 603 & 280,5 & 554 & 1058 & 1086,5 & 354 & 594 & 723 & 1066 & 1092,5 & 8085 \\
$\begin{array}{l}\text { Taux d'intérêt } \\
\text { mensuel moyen (\%) }\end{array}$ & 15,8 & 18,6 & 23 & 17,4 & 17,2 & 5,8 & 12,4 & 14,8 & 7,9 & 8,8 & 5,3 & 12 \\
& & & & & & & & & & & &
\end{tabular}

Nous avons donc effectué deux types d'études : l'une sur le taux d'intérêt sur l'ensemble du cycle, l'autre à l'intérieur de chaque réunion.

\section{- Les taux d'intérêt sur l'ensemble du cycle}

Nous avons mené une série de calculs et de tests, tant les résultats étaient ambigus.

1) Un graphique du taux moyen d'intérêt selon le rang de la réunion suggère une liaison linéaire négative, et non la constance attendue.

GRAPHIQUE 2

Évolution du taux d'intérêt des petits lots durant le cycle

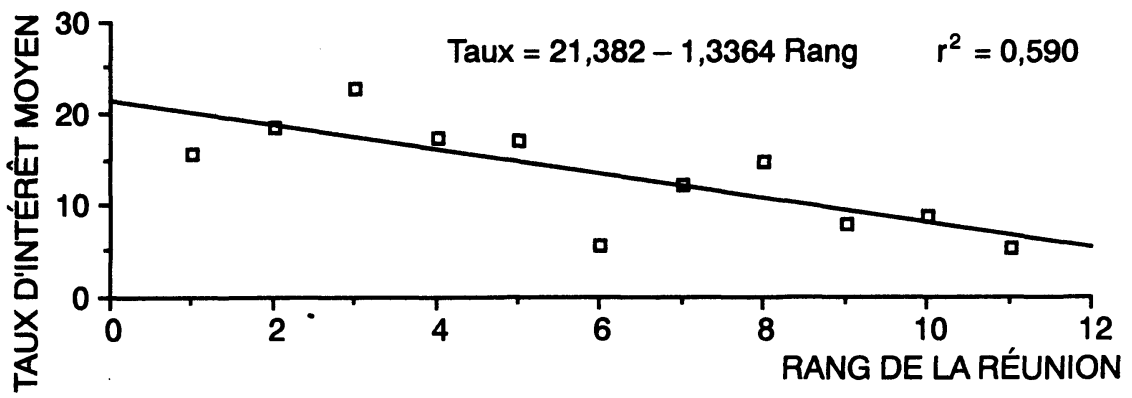

Le calcul confirme la décroissance du taux d'intérêt des petits lots selon l'avancement de l'ensemble du cycle :

taux moyen d'intérêt $=-1,336$ rang de la réunion $+21,38 \quad r=0,762$ 
Pondéré par les sommes prêtées, l'ajustement donne sensiblement le même résultat $(r=0,75)$, mais la pente est plus faible ${ }^{17}$. Enfin, une corrélation de Spearman entre les rangs des réunions et les rangs des taux d'intérêt n'apporte que peu $(r=0,79)$. Par contre, si l'on sépare, aux fins d'analyse, les 5 premières réunions des 6 dernières, on constate, pour les premières, une pente positive du taux d'intérêt moyen par réunion en fonction du rang de la réunion (mais non significativement différente de zéro) et une pente négative pour les secondes (non significativement différente de zéro).

2) Un test de comparaison des taux d'intérêt moyens a été effectué entre les 6 premières réunions et les 5 dernières : aucune différence significative n'a pu être relevée. Mais le même test, effectué sur les 5 premières réunions face aux 6 dernières, indique une différence hautement significative (à près de $1 \%$ ) montrant que les taux moyens des 5 premières réunions sont sensiblement plus élevés que lors des 6 dernières. Compte tenu des sommes prêtées, le premier test paraît plus indiqué ; le deuxième semble plus pertinent quand on prend en compte la somme des intérêts.

3) Devant ces ambiguïtés, nous avons effectué sur l'ensemble des 11 réunions une analyse de variance sur les taux de chaque prêt en fonction du rang de la réunion qui laisse apparaître une différence très significative $(1 \%)$ : les taux varient selon les réunions. Mais si l'on se contente des 10 premières réunions en faisant remarquer qu'à la $11^{\mathrm{e}}$ réunion un accord tacite semble s'être fait pour ne pas surenchérir, la différence n'est significative qu'à $2,5 \%$ environ.

En conclusion, il semble que l'hypothèse de base de la rationalité financière et économique se trouve infirmée, c'est-à-dire qu'il se révèle, contrairement à ce qui était attendu, que les taux d'intérêt moyens des petits lots baissent au fur et à mesure que progresse le cycle. Mais cette affirmation manque de vigueur.

\section{- Les taux d'intérêt des petits lots de chaque réunion}

Il peut sembler logique de penser que les premiers petits lots de chaque réunion soient plus demandés que les derniers, le montant des enchères mesurant l'intensité du besoin de crédit. Nous avons mené deux vastes études pour confirmer cette hypothèse :

17. Taux moyen d'intérêt $=-1,266$ Rang de la réunion $+20,28$. 
- Une comparaison des taux d'intérêt des prêts selon qu'ils sont situés avant ou après le taux médian. Il en ressort qu'aucune différence significative n'a pu être mise en évidence entre les premiers d'une réunion à lever un petit lot et les derniers.

- Nous avons calculé les ajustements pondérés par les sommes prêtées entre les taux d'intérêt et le rang du petit lot de la réunion. Là encore, aucune différence significative n'a pu être mise en évidence entre les premiers et les derniers.

\section{Conclusion}

La levée des petits lots ne répond pas aux hypothèses de base de la rationalité financière. En effet, on peut montrer d'une part que le taux d'intérêt du crédit à un mois baisse au cours du cycle et, d'autre part, qu'à l'intérieur d'une réunion, il n'existe pas de différence significative selon l'urgence présumée des besoins.

\subsection{Intérêt financier de la participation à la tontine}

Il est possible d'intégrer les deux approches précédentes et d'établir pour chaque individu la comptabilité complète de sa participation à la tontine. Cette comptabilité est affinée en trois temps :

- D'abord, une comptabilité concernant seulement le paiement des cotisations et la levée du lot principal.

- Ensuite, une comptabilité comprenant la prise en compte des cotisations et de la levée du lot principal, ainsi que des opérations concernant les petits lots, comptabilité qui indique, par rapport à la première, l'incidence des emprunts de petits lots et des retards de remboursement de ceux-ci qui ont entraîné des pénalités.

- Enfin, une approche globale qui intègre les cotisations et les levées des lots principaux, les opérations sur les petits lots et tous les frais de convivialité : cette procédure rend compte de l'intérêt financier global d'un individu à la participation à la tontine.

Dans toutes ces études, deux démarches sont possibles : l'une sans prise en compte du temps dans le cycle des opérations, l'autre qui l'y intègre. 


\subsubsection{L'approche intemporelle}

Pour chaque individu, on calcule le solde de sa participation à la tontine sans tenir compte du moment où les dépenses et les recettes ont lieu. Nous obtenons le tableau suivant :

Tableau 7

Solde des opérations de la tontine en fonction du rang de la levée Unité : soldes en milliers de FCFA

\begin{tabular}{|c|c|c|c|c|c|c|}
\hline \multirow[b]{2}{*}{ Réunion } & \multirow[b]{2}{*}{ Tour } & \multirow[b]{2}{*}{$\begin{array}{l}\text { Numéro } \\
\text { d'adju- } \\
\text { dicataire }\end{array}$} & \multicolumn{3}{|c|}{ Solde pour } & \multirow[b]{2}{*}{$\begin{array}{c}\text { Nature } \\
\text { de } \\
\text { l'intervenant }\end{array}$} \\
\hline & & & $\begin{array}{c}\text { Lot } \\
\text { principal }\end{array}$ & $\begin{array}{c}\text { Lot } \\
\text { principal } \\
+ \text { petits lots }\end{array}$ & $\begin{array}{c}\text { Lot } \\
\text { principal } \\
\text { + petits lots } \\
\text { + fêtes }\end{array}$ & \\
\hline 1 & 1 & 22 & $-61,5$ & $-118,5$ & $-213,5$ & 0 \\
\hline 1 & 2 & 3 & $-126,5$ & $-155,5$ & $-250,5$ & $\mathbf{P}$ \\
\hline 2 & 3 & 14 & $-166,5$ & $-166,5$ & $-261,5$ & É \\
\hline 2 & 4 & 12 & $-171,5$ & -423 & -518 & $\overline{\mathbf{R}}$ \\
\hline 2 & 5 & 15 & $-125,5$ & $-224,5$ & $-319,5$ & A \\
\hline 3 & 6 & 23 & -125 & -331 & -426 & $\mathbf{T}$ \\
\hline 3 & 7 & 20 & -100 & -139 & -234 & $\mathbf{E}$ \\
\hline 4 & 9 & 8 & 56 & 49 & -46 & $\mathbf{U}$ \\
\hline 4 & 10 & 19 & 91 & 91 & -4 & $\mathbf{R}$ \\
\hline 5 & 11 & 28 & 38,6 & $-120,1$ & $-215,1$ & \\
\hline 6 & 13 & 9 & 136 & 72,8 & 22,2 & \\
\hline 6 & 14 & 11 & 141 & 141 & 46 & F \\
\hline 7 & 15 & 13 & 133 & 71 & -24 & I \\
\hline 7 & 16 & 25 & 136 & 105 & 10 & $\mathbf{N}$ \\
\hline 7 & 17 & 21 & 140 & 122 & 27 & A \\
\hline 8 & 18 & 5 & 282 & 282 & 187 & $\mathbf{N}$ \\
\hline 8 & 20 & 24 & 86 & 86 & -9 & $\mathrm{C}$ \\
\hline 9 & 21 & 10 & 141 & 75,5 & 19,5 & I \\
\hline 9 & 22 & 7 & 141 & 141 & 46 & $\mathrm{E}$ \\
\hline 10 & 23 & 17 & 136 & 136 & 41 & $\mathbf{R}$ \\
\hline 10 & 24 & 18 & 120 & 112 & 17 & \\
\hline 11 & 25 & 27 & 113,55 & 113,55 & 18,55 & \\
\hline 12 & 26 & 26 & 113,55 & 108,55 & 13,55 & \\
\hline 12 & 27 & 1 & 141 & 141 & 46 & \\
\hline 12 & 28 & 2 & 141 & 104 & 9 & \\
\hline
\end{tabular}

Dans cette approche la plus simple de la réalité, la distinction entre les opérateurs et les financiers frappe : globalement, tous les opérateurs sont en déficit pour leur participation à la tontine, certains très lourdement (individu 
$\mathrm{n}^{\circ} 12:-518000 \mathrm{~F}$ ), d'autres légèrement (individu $\mathrm{n}^{\circ} 9:-4000 \mathrm{~F}$ ) ; par contre, parmi les financiers, la participation à la tontine se conclut de façon positive dans la plupart des cas (11 sur 15) et quand le solde est négatif, il l'est faiblement (-24000 $\mathrm{F}$ au maximum); si l'on ne compte pas les dépenses de convivialité qui représentent $95000 \mathrm{~F}$, strictement tous les financiers et deux opérateurs font une opération positive. Le rapport financier paraît donc l'argument fort quand il s'agit de participer à la tontine.

- Pour les 11 individus à solde global positif, la participation à la tontine apparaît comme une opération hautement rentable : en dehors du bénéfice financier tiré du cycle de la tontine (et qui au maximum monte à $187000 \mathrm{~F}$ dans le cas particulier d'un individu à 2 parts) chacun a droit à 12 repas et 2 fêtes.

- La deuxième situation est représentée par les 6 individus ayant un solde final négatif, mais un solde du lot principal et des petits lots positif. Pour ceux-ci, la participation à la tontine coûte une somme minime, mais donne droit à tous les bénéfices de la convivialité.

- L'argument est quelque peu différent pour les 8 individus qui ont un solde négatif important : comme nous le dit notre informateur, «il est évident que celui qui a payé $1400000 \mathrm{~F}$ à $375000 \mathrm{~F}$ ne gagne rien en fin de compte, mais il a l'argent au moment où il en avait besoin et c'est son gain ». Il est clair que cette dernière argumentation, totalement différente de la première, introduit directement le temps dans l'appréciation des avantages et des inconvénients de la participation à la tontine.

Il convient alors d'abandonner l'approche intemporelle qui ne tient pas compte de cette variable déterminante pour analyser le cycle de la tontine en tenant compte des différents moments où ont eu lieu les opérations.

\subsubsection{Analyse financière intégrant le temps}

Nous reprenons l'approche comptable progressive, afin de distinguer l'incidence des diverses opérations sur la rentabilité individuelle. Nous considérons, pour chaque individu, l'ensemble de ses dépenses et de ses recettes (aux dates données) comme une vaste opération financière dont il convient de trouver le taux de rendement interne. Les résultats des calculs sont consignés dans le tableau suivant : 


\section{TABLEAU 7}

\section{Rentabilité financière du participant en fonction du rang de la levée}

Unité : rentabilité en \% par mois - indique un gros emprunteur ( $>2$ emprunts)

\begin{tabular}{|c|c|c|c|c|c|c|}
\hline \multirow[b]{2}{*}{ Réunion } & \multirow[b]{2}{*}{ Tour } & \multirow[b]{2}{*}{$\begin{array}{l}\text { Numéro } \\
\text { d'adju- } \\
\text { dicataire }\end{array}$} & \multicolumn{3}{|c|}{ Solde pour } & \multirow[b]{2}{*}{$\begin{array}{c}\text { Nature } \\
\text { de } \\
\text { l'intervenan }\end{array}$} \\
\hline & & & $\begin{array}{c}\text { Lot } \\
\text { principal }\end{array}$ & $\begin{array}{c}\text { Lot } \\
\text { principal } \\
+ \text { petits lots }\end{array}$ & $\begin{array}{c}\text { Lot } \\
\text { principal } \\
\text { + petits lots } \\
\text { + fêtes }\end{array}$ & \\
\hline 1 & 1 & 22 & $-1,22$ & $-2,12$ & $-3,81$ & 0 \\
\hline 1 & 2 & 3 & $-1,44$ & $-1,7$ & $-2,76$ & $\mathbf{P}$ \\
\hline 2 & 3 & 14 & $-3,73$ & $-3,73$ & $-5,98$ & É \\
\hline 2 & 4 & 12 & $-3,76$ & $-6,69$ & $-8,37$ & $\mathbf{R}$ \\
\hline 2 & 5 & 15 & $-2,03$ & $-3,76$ & $-5,21$ & $\mathbf{A}$ \\
\hline 3 & 6 & 23 & $-3,48$ & $-7,2$ & $-9,6$ & $\mathrm{~T}$ \\
\hline 3 & 7 & 20 & $-2,7$ & $-3,43$ & $-5,99$ & $\mathrm{E}$ \\
\hline 4 & 9 & 8 & $-1,79$ & $-1,51$ & $-1,54$ & $\mathbf{U}$ \\
\hline 4 & 10 & 19 & $-2,78$ & $-2,78$ & $-0,13$ & $\mathbf{R}$ \\
\hline 5 & 11 & 28 & $-1,73$ & $-4,23$ & -8 & \\
\hline 6 & 13 & 9 & 20,18 & $?$ & $-2,95$ & \\
\hline 6 & 14 & 11 & 20,47 & 20,47 & 12,51 & F \\
\hline 7 & 15 & 13 & 10,05 & 7,95 & ? & I \\
\hline 7 & 16 & 25 & 10,2 & 9,18 & 1,25 & $\mathbf{N}$ \\
\hline 7 & 17 & 21 & 10,4 & 10,88 & 3,57 & A \\
\hline 8 & 18 & 5 & 3,55 & 3,55 & 2,31 & $\mathbf{N}$ \\
\hline 8 & 20 & 24 & 4 & 4 & $-0,44$ & $\mathrm{C}$ \\
\hline 9 & 21 & 10 & 4,1 & 2,65 & $-0,68$ & I \\
\hline 9 & 22 & 7 & 4,1 & 4,1 & 1,31 & $\mathrm{E}$ \\
\hline 10 & 23 & 17 & 2,95 & 2,95 & 0,94 & $\mathbf{R}$ \\
\hline 10 & 24 & 18 & 2,63 & 2,52 & 0,37 & \\
\hline 11 & 25 & 27 & 2,26 & 2,26 & 0,45 & \\
\hline 12 & 26 & 26 & 1,84 & 1,79 & 0,29 & \\
\hline 12 & 27 & 1 & 2 & 2 & 0,62 & \\
\hline 12 & 28 & 2 & 2 & 1,46 & 0,12 & \\
\hline
\end{tabular}

Les opérateurs se singularisent en ayant tous des taux de rendement négatifs pour leur participation à la tontine, résultat logique provenant du fait que pour chacun la participation à la tontine se solde par un déficit ${ }^{18}$. Ce rendement négatif évolue entre $-0,13 \%$ et $-9,6 \%$ par mois avec une moyenne de

18. Un taux de rendement négatif signifie que ces individus sont en déficit si l'on considère la somme arithmétique de leurs apports et de leurs levées : de ce fait, ils sont emprunteurs nets et le taux de rendement négatif est le taux auquel ils auraient pu emprunter l'argent de l'opération. 
$-5,13 \%$. Ce rendement négatif semble largement influencé par le fait d'être ou non un gros emprunteur. Si l'on reprend la distinction faite plus haut, les gros emprunteurs ( $>2$ emprunts de petits lots) connaissent une rentabilité de $-6,24 \%$ alors que les petits emprunteurs ( $\leq 2$ emprunts) se limitent à $-2,55 \%$. Toutefois, pour importante qu'apparaisse la différence, elle n'est significative qu'à environ $8 \%$ avec le test $t$ de Student (mais à $5 \%$ avec le test de Mann et Whithney).

Pour ce qui concerne les financiers, la première idée est de faire la même comparaison entre gros emprunteurs et petits emprunteurs. Mais cette fois, la différence de comportement n'est pas significative du tout (et irait plutôt dans l'autre sens) : ce n'est donc pas le fait d'être gros ou petit emprunteur de petits lots qui compte pour l'explication du taux de rentabilité de la participation à la tontine. Le facteur clé de la rentabilité pour les financiers semble être le rang de la réunion au cours de laquelle a eu lieu la levée du lot principal : il faut y procéder le plus tôt possible et ensuite s'abstenir d'emprunter des petits lots. Cette stratégie a été suivie par le $n^{\circ} 11$; par contre, l'individu $n^{\circ} 1$ a beaucoup perdu en rentabilité en n'enchérissant pas pour le lot principal plus tôt : une réunion plus tôt et pour une enchère minime, il gagnait $0,5 \%$ de rentabilité mensuelle; deux réunions plus tôt, il gagnait pratiquement $1 \%$. Mais il est évident que si un tel raisonnement, intégrant totalement le temps, était tenu par tous les participants, on découvrirait un lissage parfait de la courbe de rentabilité interne, les enchères formant le facteur de lissage.

\section{Conclusion}

En 11 mois, 25 personnes (comptant 28 parts) mettent en mouvement une somme de $28 \times 28 \times 50000 \mathrm{~F}$, soit 39,2 millions de francs ${ }^{19}$; cette somme représente 1,4 million par part. Ce montant apparaît comme particulièrement important, tant au regard des disponibilités individuelles que des revenus locaux. Dans le cas des opérateurs, on peut penser que la tontine a servi à diverses opérations économiques, mais je n'ai pas de renseignements sur celles-ci. À l'inverse, dans le cas des financiers (15 membres sur 25 représentant 16 parts sur 28), on peut poser l'hypothèse que la participation à la tontine correspond à une opération de placement financier assez lucratif ou bien à une consommation différée en vue d'un gros achat, les deux n'étant pas exclusifs, bien au contraire. Il est probable qu'une fraction des sommes mises en mouvement ne corresponde

19. En fait légèrement plus, car à la fin de la dernière réunion, il reste en caisse un reliquat de $309170 \mathrm{~F}$, soit environ $11000 \mathrm{~F}$ par part, que les membres se partagent. 
pas à une épargne de la période, mais à l'utilisation d'une épargne préalable : quelque importants que soient les revenus de cette élite tontinière, quelques individus n'arrivent pas à épargner $100000 \mathrm{~F}$ par mois (voire $200000 \mathrm{~F}$ pour ceux qui disposent de deux parts) : il leur faut donc puiser dans une épargne antérieure ; éventuellement, ils peuvent aussi différer cette ponction en levant très tôt dans le cycle ou en empruntant de nombreux petits lots : le problème financier de tels participants devient alors relativement facile à gérer pour eux, même si leur participation à la tontine leur coûte de l'argent.

Parmi les participants à cette tontine, certains ont obtenu de nombreux crédits à un taux abordable ou faible dans les conditions locales. Réciproquement, d'autres ont effectué, par le truchement de la tontine, une opération financière intéressante rapportant 2 ou $3 \%$ par mois! Tous ont bénéficié des avantages de la convivialité et ceux-ci sont conséquents. Il n'est donc pas étonnant de constater l'enthousiasme des participants pour ce genre de tontine : ceux qui ont bénéficié du crédit insistent sur le fait que dans le domaine formel une banque aurait tergiversé longtemps, exigé de nombreuses formalités, sans qu'à aucun moment ils n'aient la certitude d'obtenir le prêt demandé à la date prévue; ailleurs que dans le domaine informel, les prêts inter-individus sont beaucoup plus chers tout en ne portant que sur des sommes très faibles; de leur côté, les financiers insistent sur le rapport particulièrement élevé de cette opération qui est sans commune mesure avec ce que peuvent offrir la participation à une entreprise ou le dépôt de l'argent en banque. De plus, des erreurs de calcul amplifient chez les financiers les avantages perçus et diminuent chez les opérateurs le coût du crédit ${ }^{20}$. Tous soulignent, qu'en dehors des avantages financiers, il convient de compter la convivialité au titre des avantages de la participation à la tontine.

De nombreux auteurs, et en particulier N. Mourgues, ont affirmé que les tontines constituaient un système de prêts et d'emprunts dans lequel la variable temps n'est pas prise en compte. Cela me semble totalement exact dans le cas des tontines mutuelles. Par contre, avec les tontines à enchères, cette mesure de la préférence intertemporelle me paraît particulièrement précise. Les enchères adaptent les emprunts et les prêts à cette préférence sans autre technique de rationnement que le prix. Du point de vue théorique, il me semble donc que l'on peut s'opposer totalement à $\mathrm{N}$. Mourgues qui affirme que «même les tontines à enchères fonctionnent sans que la variable temps soit prise en compte. Certes, dans les tontines à enchères le droit de lever est payant. Mais ce droit ne peut se traduire par un taux d'actualisation pour distinguer la préférence du

20. Même notre correspondant se trompe dans le décompte du nombre de réunions et du rapport financier de sa participation à la tontine. 
présent au futur ${ }^{21}$. On peut toutefois admettre que dans la pratique, la prise en compte du temps est loin d'être totale. En effet, si l'hypothèse que dans une tontine à enchères le temps est une variable essentielle semble bien vérifiée lorsque l'on considère les enchères du lot principal (car celles-ci décroissent avec le rang de la réunion), on peut objecter d'une part qu'à l'intérieur d'une réunion donnée, les enchères par tour ne diffèrent pas significativement (tant pour les lots principaux que pour les petits lots) et, d'autre part, qu'un certain nombre d'individus semble n'avoir pas compris - tout en appartenant aux financiers - qu'il est plus rentable d'enchérir très légèrement pour lever le lot principal beaucoup plus tôt : c'est donc qu'une partie essentielle de l'appréhension du temps leur a échappé. Enfin, on peut remarquer que même en ce qui concerne les petits lots, on constate une baisse progressive du taux d'intérêt au cours du cycle, alors que les crédits sont toujours du même type.

Ces deux derniers points sont susceptibles d'une explication où le temps n'intervient pas : faisons l'hypothèse que les participants « pauvres » à la tontine sont obligés, pour tenir leurs engagements, soit de lever tôt le lot principal, soit de solliciter de nombreux petits lots ; avec le désintéressement successif de ces individus, la pression de la demande concernant le lot principal baisse en même temps que celle concernant les petits lots. Si cette hypothèse s'avérait fondée, cela diminuerait en même temps la portée de la décroissance des enchères du lot principal laquelle dépendrait non de la préférence intertemporelle, mais de celle-ci via la « pauvreté » de certains adjudicataires.

On peut aussi penser que les enchères intègrent un élément d'excitation et d'irrationalité : en effet, comment expliquer autrement qu'à la $8^{\mathrm{e}}$ réunion, le deuxième adjudicataire emporte les enchères à $125000 \mathrm{~F}$ alors que le premier emporte le lot aux enchères minimales de $70000 \mathrm{~F}$ : le deuxième adjudicataire aurait pu lever le premier lot à $71000 \mathrm{~F}$, mais la passion l'a conduit à payer $125000 \mathrm{~F}$ ! L'intégration de cet élément passionnel étant impossible, et variant de réunion en réunion, les conclusions logiques manquent naturellement de force.

21. N. Mourgues, «Les tontines. Analyse des mécanismes financiers», Table ronde sur les circuits informels de financement des entreprises, Bangui, mai 1985, publié par l'Institut orléanais de finance. 